\title{
Isotope Effect as a Probe of the Role of Phonons in Conventional and High Temperature Superconductors
}

\author{
Manzoor A. Malik*, Bilal A. Malik
}

Department of Phy sics, University of Kashmir, Srinagar, 190006, J\&K, India

\begin{abstract}
We review the ro le of phonons in the mechanism of both conventional and high temperature superconductivity. We find that while on one hand most of the conventional superconductors fall within the framework of the BCS phonon-mediated pairing, on the other hand the complexity of the structural properties of High $T_{C}$ superconductors allows determination of only the partial (oxygen) isotope effect, with values ranging from megligibly s mall to even higher than BCS prediction. In either case, the study of isotope shift is important in its own right. We arrive at the conclusion that while phonons are central to explaining the mechanis m of conventional superconductivity, their ro le should be taken into account for exp laining High $\mathrm{T}_{\mathrm{C}}$ superconductivity, as well.
\end{abstract}

Keywords Superconductivity, Conventional, High Temperature, Isotope Effect, Mechanism

\section{Introduction}

One of the most important properties of metals is that their electrical resistivity decreases with the decrease of temperature. Investigation of the electrical properties of metals at low temperatures led the Dutch Physicist Heike Kamerlingh Onnes and his assistant Gilles Holst in 1911 to discover, the phenomenon of superconductivity at $4.2 \mathrm{~K}$ in mercury[1, 2]. The discovery of superconductivity was one of the most important experimental finding in low temperature physics of the early 20th century. Since then, tremendous theoretical and experimental efforts have been made to develop the correct theory for explaining underlying mechanism responsible for superconductivity. In the history of superconductivity, different theories have been proposed from time to time. We can broadly categorize these theoretical efforts into two types: Macroscopic and Microscopic theories. The macroscopic theories like Meissner effect[3], London equations [4], Ginsburg-Landau theory[5], do fairly exp lain some properties of superconductors but were inadequate to expla in the underlying mechanis m of superconductivity.

The first microscopic theory of superconductivity was put forth by Bardeen, Cooper and Schrieffer in 1957[6]. For conventional superconductors, this theory satisfactorily explains the properties like Penetration Depth, the Energy Gap, Coherence Length, Isotope Effect and other properties as summarized in[7,8]. As BCS theory could explain almost all the properties of conventional superconductors, supported

* Corresponding author:

mmalik@kashmiruniversity.ac.in (Manzoor A. Malik)

Published online at http://journal.sapub.org/ajcmp

Copyright (C) 2012 Scientific \& Academic Publishing. All Rights Reserved by fairly good agreement between theory and experiment, it was considered to be ultimate theory for the mechanism of superconductivity.

However, the discovery of high temperature superconductivity[9], particularly above the liquid nitrogen temperature[10-13] posed serious challenges to the validity of BCS theory. Despite being the most active area of research and its limitless scope for applications, the mechanism remains elusive, owing to the unusual nature of properties exhibited by these high $T_{C}$ materials, particularly cuprates. The properties like very small or no isotope shift, high transition temperature, short coherence length, low density of states, low carrier concentration, electric and magnetic anisotropies are markedly different from those for the conventional low temperature superconductors. These discrepancies which could not be accommodated with in the original BCS forma lis $\mathrm{m}$ led res earchers to search for alternative theories of high temperature superconductivity including modification to the original BCS framework so as to somehow account for these unusual properties of High $T_{C}$ superconductors.

Among the various properties of superconductors, the one central to the development of BCS theory was the observation of Isotope Effect in superconductors. In conventional superconductors, it was found that transition temperature is inversely proportional to the square root of the is otopic mass ( $T_{C} \propto \mathbf{M}^{-\alpha}$ where $\alpha \approx 0.5$ ). Isotopes being chemically identical, a change in $T_{C}$ on substituting one isotope for other implies that the lattice vibrations are somehow important for superconductivity. This was the basis for the phonon mediated pairing interaction envisaged in the BCS theory $v$ ia the formation of cooper pairs [14].

Certain conventional superconductors have is otope effect exponent equal to the predicted value of BCS theory and lot 
of materials have small but appreciable deviations from the BCS value, which for practical purposes can be treated within the BCS framework. But there are conventional superconductors which have is otope effect much larger than BCS value which though may be exceptions, raise serious challenge against theory $[15,16]$.

On the other hand, the story of high temperature superconductors is interesting and challenging. The study of oxygen isotope effect has been carried out in almost all cuprates. For some family of cuprates, the oxygen isotope exponent (OIE) is very s mall than BCS prediction[17]. This suggests that phonons may not be playing any role in the occurrence of superconductivity in cuprates. However, for a particular family of doped cuprates, the oxygen isotope exponents (OIE) increase with decreasing $T_{C}$ and can be even larger than BCS value[18]. Such an anomalously large OIE imply that phonons have a definite role in the superconductivity.

Any correct theory for explaining the mechanism of superconductivity, in particular, for high $T_{C}$ superconductors has to account for both small OIE in optimally-doped samples and anomalously large OIE in the underdoped samples.

Motivated by the central role that is otope effect plays in explaining conventional superconductivity and the recent findings that phonons may be important for high $T_{C}$ superconductors, we intend, in this review, to put together the efforts of various groups in trying to explain both conventional and high $T_{C}$ superconductivity.

\section{Isotope Effect in Conventional Superconductors}

Is otope effect studies have been vital to the development of the microscopic theory of superconductivity. The first piece of evidence about the existence of isotope effect in mercury was reported in 1950 by Maxwell[19] and independently by Reynold et al.[20]. They found that the critical temperature $T_{C}$ of mercury varies as an inverse function of is otopic mass. The argument that is otope mass enters into the formation of the superconducting phase implies that superconductivity is not purely electronic in origin. In the same year, Frohlich[21] proposed that same electron lattice interaction responsible for the scattering of conduction electrons by lattice vibrations provides a necessary glue between electrons. Frohlich's theory got a strong base from the experimental observation of isotope effect and played a key role in understanding microscopic mechanism of superconductivity. In 1956, Cooper[14] demonstrated that a pair of electrons can interact above a Fermi surface with an interaction of a kind that might be expected due to phonon and screened coulomb fields. If there is a net attraction between the electrons, it turns out that they can form bound states, though their energy is larger than zero. The properties of non-interacting system of bound pairs are very suggestive of those which could produce superconducting state. However, the existence of electron pairs does not necessarily imply a phonon mediated pairing. Indeed Bose condensation, as considered by Schafroth[22] is also a possible mechanism for superconductivity, but unfortunately the model was not able to exp lain one of the important experimentally observed property of is otope effect discovered earlier by Maxwell and Reynold. Finally in 1957 Bardeen, Cooper and Schrieffer[6], in view of experimental observation of isotope effect in mercury discovered by Maxwe11[19] and Reynold[20] followed by theoretical consideration of Frolich [21] and inadequacy of Schafroths condensation[22], put forward first successful microscopic theory, which explains most of the physical properties observed in conventional superconductors.

Within the framework of BCS microscopic theory, one has relation between transition temperature $T_{C}$, typical phonon frequency $\omega$ (e.g Debye frequency) and interaction strength $N\left(E_{f}\right) V$ as

$$
K_{B} T_{C}=1.13 \hbar \omega \exp \left(-\frac{1}{\mathrm{VN}\left(\mathrm{E}_{\mathrm{f}}\right)}\right)
$$

Where $\mathrm{V}$ is the pairing potential arising fro $\mathrm{m}$ the electron phonon interaction, $N\left(E_{f}\right)$ is the electronic density of states at Fermi surface and $K_{B}$ is the Boltzmann constant. Equation (1) is considered as one of the most significant and influential prediction of BCS theory. In the harmonic approximation both $\mathrm{V}$ and $N\left(E_{f}\right)$ are independent of the ionic mass, while characteristic frequency $\omega$ can be expressed as

$$
\omega \propto \frac{1}{\sqrt{\mathbf{M}}}
$$

where $\mathrm{M}$ is the ionic mass. By combining equations (1) and (2), one can deduce that if the product $V N\left(E_{f}\right)$ is increased, then $T_{C}$ will rise. Further, the frequency $\omega$ can be enhanced using lighter elements, that in turn enhances the $T_{C}$ of corresponding superconductors. On the basis of equations (1) and (2), BCS predicted a maximum $T_{C}$ of around $30 \mathrm{~K}$, which did explain the transition temperatures of conventional superconductors with a record high $T_{C}$ of $23.2 \mathrm{~K}$ in an alloy of $\mathrm{Nb}$ and $\mathrm{Ge}[23,24]$.

On the basis of electron phonon mechanism, the $T_{C}$ can be described by the following relation

$$
\mathrm{T}_{\mathrm{C}}=A M^{-\alpha}
$$

Where $\mathrm{A}$ is a constant, $\mathrm{M}$ is the mass element substituted by its isotope and $\alpha$ is the is otope effect coefficient, which is defined by the following relation

$$
\boldsymbol{\alpha}=-\frac{\partial \ln T_{C}}{\partial \ln M} \approx-\frac{M}{T_{C}} \frac{\Delta T_{C}}{\Delta M}
$$

where $\Delta M$ is mass difference between two isotopes and $\Delta T_{C}$ is the shift of critical temperature by the substitution of is otopic mass.

With the help of equations (1) and (2) we can conclude that in BCS theory $T_{C}$ is inversely proportional to square root of masses of the is otopic elements i.e. the is otope effect coefficient predicted by BCS theory has the value $\alpha \approx 0.5$, which is in excellent agreement with the reported isotopic effect in many metals as shown in the first nine entries in table 1.

From table 1, it follows that for many superconducting materials $\alpha$ is indeed close to the BCS value of 0.5 . As 
shown in table 1 , the inverse isotope effect $(\alpha<0)$ has also been reported e.g in uranium $\alpha=-2$. A reversal of isotope effect was also observed[48] in $\operatorname{PdH}(D)$ system, where the transition temperature increases on substitution of deuterium for Hydrogen. This effect was qualitatively exp lained[49] as resulting from differences in the electronic structure arising from anharmonicity in the zero point motion of hydrogen isotopes. The existence of reversal isotope effect demonstrates that while discussing isotope effect one has to take into account the band structure as well. Besides, there are many organic superconductors in which the inverse isotope effect has been observed[50]. Thus, isotope effect is not a universal phenomenon and can be absent even in conventional superconductors (e.g in $\mathrm{Zr}, \alpha=0 \pm 0.05$ and in $\mathrm{Ru}, \alpha=$ $0 \pm 0.15$ ). The obvious discrepancy between observation and theory demonstrates limitations of simplified BCS model. Nevertheless, this BCS theory based on phonon mediated mechanis $m$ has played a decisive role in showing path to the correct theory of superconductivity in most of metals and alloys. Further, it also served as the basis for more sophisticated theories of superconductivity.

Table 1. Critical Temperature $T_{C}$ and Isotope effect exponent $(\alpha)$

\begin{tabular}{|c|c|c|c|c|}
\hline $\begin{array}{c}\text { Super- } \\
\text { con-ductor }\end{array}$ & $\begin{array}{c}\mathrm{T}_{\mathrm{C}} \\
(\mathrm{k})\end{array}$ & Ref. & $\alpha$ & Ref. \\
\hline $\mathrm{Hg}$ & 4.15 & {$[25]$} & $0.5 \pm 0.03$ & {$[19,28]$} \\
\hline $\mathrm{Tl}$ & 2.39 & {$[25]$} & $0.5 \pm 0.1$ & {$[19,28,29]$} \\
\hline $\mathrm{Cd}$ & 0.56 & {$[26]$} & $0.5 \pm 0.1$ & {$[30]$} \\
\hline $\mathrm{Pb}$ & 7.2 & {$[7]$} & 0.48 & {$[7]$} \\
\hline $\mathrm{Sn}$ & 3.72 & {$[26]$} & 0.46 & {$[27]$} \\
\hline $\mathrm{Re}$ & 1.69 & {$[25]$} & 0.38 & {$[31]$} \\
\hline $\mathrm{Mo}$ & 0.92 & {$[25]$} & $0.37 \pm 0.04$ & {$[32,33]$} \\
\hline $\mathrm{Zn}$ & 0.87 & {$[25]$} & $0.30 \pm 0.01$ & {$[34]$} \\
\hline $\mathrm{MgB} 2$ & 39 & {$[27]$} & 0.3 & {$[35,36]$} \\
\hline $\mathrm{Os}$ & 0.65 & {$[25]$} & $0.21 \pm 0.05$ & {$[37,38]$} \\
\hline $\mathrm{Zr}$ & 0.54 & {$[25]$} & $0 \pm 0.05$ & {$[38]$} \\
\hline $\mathrm{Ru}$ & 0.51 & {$[25]$} & $0 \pm 0.15$ & {$[37,39-42]$} \\
\hline $\mathrm{U}$ & 0.68 & {$[25]$} & -2 & {$[43]$} \\
\hline $\mathrm{K}_{3} \mathrm{C}_{60}$ & 19.5 & {$[27]$} & 0.37 orl 14 & {$[16,44-47]$} \\
\hline $\mathrm{Hg}^{200.6}$ & 4.156 & {$[15]$} & 2.896 & {$[15]$} \\
\hline
\end{tabular}

Though the BCS theory did not completely succeed in explaining isotope effect in superconductors, but it paved the way for a deeper understanding of electron- phonon interaction. In the limit of strong coupling, Eliashberg model[51], the electron phonon coupling constant $\lambda_{e p}$ is related to spectral function $\alpha^{2}(\omega) \mathrm{F}(\omega)$, as defined by the product of phonon density of states $F(\omega)$ and the frequency dependent average electron phonon interaction $\alpha^{2}(\omega)$. Eliashberg's equations were solved by McMillan[52] by taking into account the coulomb repulsion between electrons, which was one of the obvious reas on for the discrepancy between theory and experimental observations and the retarded nature of phonon induced interaction:

$$
K_{B} T_{C}=\frac{\hbar \omega}{1.45} \exp \left(\frac{-1.04\left(1+\lambda_{e p}\right)}{\lambda_{e p}-\mu^{*}\left(1+0.62 \lambda_{e p}\right)}\right)
$$

where $\mu^{*}$ is the effective coulomb repulsion. Under $\lambda_{e p} \ll 1$ i.e weak interaction, it reduces to equation (1) where $\lambda_{e p}-\mu^{*}$ substitutes the role of $N\left(E_{f}\right) V$. As a result, in strong-coupling superconductors, the isotope effect exponent has no longer a universal value and is given by

$$
\alpha=\frac{1}{2}\left[1-\left(\mu^{*} \log \frac{\hbar \omega}{1.45 K_{B} T_{C}}\right)^{2}\left(\frac{1+0.62 \lambda_{e p}}{1+\lambda_{e p}}\right)\right]
$$

Above equation suggest a negative or small isotope exponent is also possible within the framework of the Eliashberg theory. Thus, the existence of small isotope exponent is still consistent with phonon-mediated pairing mechanis $m$.

\section{Isotope Effect in High $T_{C}$ Cuprates Superconductors}

The discovery of superconductivity in $\mathrm{La}_{2-\mathrm{x}} \mathrm{Ba}_{\mathrm{x}} \mathrm{CuO}_{4}$ by Bednorz and Muller in 1986[9] prompted a burst of experimental and theoretical investigations in these systems in order to clarify the microscopic pairing mechanis $\mathrm{m}$ for high temperature superconductivity. However, the mechanism is still highly controversial because the opinions as regards the role of electron phonon interaction vary widely[53-55]. A lso, the unprecedentedly high transition temperatures in some cuprates were difficult to reconcile with the conventional phonon mediated mechanism which could account for a maximum $T_{C}$ of $30 \mathrm{~K}[52]$. Existence of short coherence length raises further challenges against BCS theory.

Cuprates have a complex structure as compared to elemental superconductors and essentially have layered character, confirmed by the anisotropy of their properties. Usually cuprates consist of four layers: (a) Conducting $\mathrm{DO}_{2}$ layer (i.e $\mathrm{CuO}_{2}$ planes), (b) $\mathrm{C}$ separating Layer typically like $\mathrm{Ca}$ or $\mathrm{Y}$, (c) $\mathrm{BO}$ bridging layer typically like $\mathrm{BaO}, \mathrm{LaO}$ or $\mathrm{SrO}$, and (d) $\mathrm{AO}$ additional layer like $\mathrm{BiO}, \mathrm{HgO}$ or TlO. Superconductivity is believed to take place in $\mathrm{CuO}_{2}$ planes which are present in all high $T_{C}$ superconductors, but the number of $\mathrm{CuO}_{2}$ planes varies among the different families of cuprates, ranging from a single-layer up to an infinite-layer structure. Thus, the existence of layer structure introduces complexity in the investigation of isotope effect in cuprates.

Hence, if a superconductor is composed of different elements like cuprates, one defines a partial isotope coefficient as

$$
\alpha_{i}=-\frac{\Delta T_{C}^{i}}{T_{C}} \frac{M}{\Delta M_{i}}
$$

where index i denotes a constituent with mass $\mathrm{M}_{\mathrm{i}}$ (e.g. oxygen). At first sight, it seems the magnitude of $\alpha_{i}$ may provide insight into which phonons are important in the occurrence of superconductivity. However, small or even vanishing partial isotope exponent do not imply that corresponding constituent is not important in the occurrence of superconductivity. In that case, one should also see the effect of isotope substitution on other parameters like effective supercarrier mass $\mathrm{m}^{*}$ and supercarrier density $\mathrm{n}_{\mathrm{s}}$ for making any decision on the role of lattice vibrations. The later effects may provide crucial information about role of phonons in understanding the physics of high $T_{C}$ superconductors. The 
studies related to isotope effect have been carried out in all well known cuprates and comprehensive review is given by Frank[17]. So far, most of the studies concern the oxygen isotope shift by replacing ${ }^{16} \mathrm{O}$ with ${ }^{18} \mathrm{O}$ partly because experimental procedures are simple and reliable [56]. Now, it is generally accepted that the cuprates doped for maximum $T_{C}$ exhibit a small but clearly non-vanishing oxygen isotope exponent $\alpha_{o}$. This is true for all the different families of doped cuprates. Table 2, shows results of oxygen isotope effect for some typical families of cuprate superconductors.

Table 2. Oxygen Isotope Effect Exponent of Cuprates

\begin{tabular}{|c|c|c|c|}
\hline Superconductor & $\mathrm{T}_{\mathrm{C}}(\mathrm{k})$ & $\begin{array}{c}\text { Oxygen Isotope } \\
\text { Effect } \\
\text { Exponent }\left(\boldsymbol{\alpha}_{0}\right)\end{array}$ & Ref. \\
\hline$(\mathrm{Ba}, \mathrm{K}) \mathrm{BiO}_{3}$ & $28-35$ & 0.6 & {$[57]$} \\
\hline $\mathrm{Ba}\left(\mathrm{Pb}, \mathrm{Bi}_{3} \mathrm{O}_{3}\right.$ & $10-15$ & 0.25 & {$[57]$} \\
\hline $\mathrm{La}_{214}$ & $30-36$ & $0.15-0.20$ & {$[57]$} \\
\hline $\mathrm{Bi}_{2} \mathrm{Sr}_{2} \mathrm{CaCu}_{2} \mathrm{O}_{8+\mathrm{y}}$ & 75 & $0.03-0.05$ & {$[17]$} \\
\hline $\mathrm{YBa}_{2} \mathrm{Cu}_{4} \mathrm{O}_{8}$ & 79.5 & $0.06-0.08$ & {$[17]$} \\
\hline $\mathrm{Tl}_{2} \mathrm{Ca}_{2} \mathrm{BaCu}_{3} \mathrm{O}_{10}$ & 121 & $0 \pm 0.12$ & {$[17]$} \\
\hline $\mathrm{Nd}_{1.85} \mathrm{Ce}_{0.15} \mathrm{CuO}_{4}$ & 24.5 & $<0.05$ & {$[17]$} \\
\hline
\end{tabular}

As is obvious from the table, electron-phonon interaction appears to be central to the superconducting pairing in $(\mathrm{Ba}$, K) $\mathrm{BiO}_{3}, \mathrm{Ba}(\mathrm{Pb}, \mathrm{Bi}) \mathrm{O}_{3}$ and $\mathrm{La}_{214}$ materials. In fact, for $\mathrm{La}_{2-\mathrm{x}}$ $\mathrm{Sr}_{\mathrm{x}} \mathrm{CuO}_{4}$ Crawford et al[18] have shown that for $\mathrm{Sr}$ concentration of $\mathrm{x}=0.125$, the oxygen isotope effect (OIE) shows a pronounced maximum of $\alpha_{o} \approx 0.85$ dropping to $\alpha_{o} \approx 0.10$ for the optimal $\mathrm{Sr}$ concentration of $\mathrm{x}=0.15$. A similar behavior is shown by $\mathrm{La}_{2-\mathrm{x}} \mathrm{Ba}_{\mathrm{x}} \mathrm{CuO}_{4}[58]$. These observations of anoma lously large OIE imply phonons play a crucial role in occurrence of high $T_{C}$ superconductivity just as they do for conventional superconductors. For the rest of the compounds in Table 2, the oxygen isotope exponents (OIE's) are similar in magnitude and remain small. This small OIE observed in the optimally-doped cuprates sugges ts that phonons may not have any role or may just be like a supporting actor in the mechanism of high $T_{C}$ superconductivity. This observation led to many exotic non-phonon mediated pairing mechanis $m[59]$. The studies of the dependence of oxygen isotope effect have been carried out in all cuprate system[18,60-63]. From these observations, it follows that any correct theory of high $T_{C}$ superconductivity ought to be consistent with both small OIE for optimally-doped samples and anomalously large OIE in the underdoped samples.

\section{Conclusions}

The studies of is otope substitution can be utilized as a very effective candidate in revealing the underlying pairing mechanis m of superconductivity. We have discussed is otope substitution effect in both conventional and unconventional superconductors. Measurements of the is otope effect on the transition temperature $T_{C}$ provides a key experimental evidence for phonon-mediated pairing, supported strongly by the BCS theory of superconductivity in most conventional superconductors. There are some conventional supercon- ductors which have a very small isotope effect, but can be treated within the framework of Eliashberg theory and thus still be consistent with phonon mediated pairing mechanism. Therefore, we can conclude that superconductivity in conventional is not of purely electronic origin but phonons have an obvious role in the occurrence of superconductivity.

However, the situation in high $T_{C}$ cuprates is very interesting. In optimally doped cuprates, the OIE is very small suggesting that phonons might not have any role in the occurrence of high $T_{C}$ superconductivity. However, for a particular family of doped cuprates the OIE increases with decreasing $T_{C}$, and can even be larger than BCS prediction. Thus the existence of strong OIE implies phonons play an important role in high temperature superconductivity, as well. Any correct theory for describing the physics of high $T_{C}$ superconductors must be consistent with small OIE in optimally doped samples and large OIE in underdoped samples. Although, the interest in phonons (as mediators) in explaining high $T_{C}$ superconductivity has waxed and waned, the current findings suggest that any theory of high $T_{C}$ cannot ignore the role of phonons.

High temperature superconductivity, however, raises serious challenges to the phonon only mechanism particularly, because of observation of short coherence length, small or no isotope shift and high $\mathrm{T}_{\mathrm{C}}$. Many scientists opined that BCS theory may not work for high $\mathrm{T}_{\mathrm{C}}$ superconductors, though the situation has changed a bit now. In fact for organic and heavy fermion superconductors BCS model had a lready been ruled out. As an alternative to phonon almost all elementary excitation of solids were proposed as mediators of electron pairs. However, none of them could satisfactorily expla in the basic feature of high $\mathrm{T}_{\mathrm{C}}$ superconductors. There are many reasons to believe that electron-phonon interaction may still be behind high $\mathrm{T}_{\mathrm{C}}$ superconductivity or spin fluctuations may hold the key. In a recent paper[64], it is demonstrated that bosonic excitations of the electronic origin may be the most favoured candidates for the formation of the high $\mathrm{T}_{\mathrm{C}}$ superconducting state. The fact that high $\mathrm{T}_{\mathrm{C}}$ superconductors exhibit strange and complex properties difficult to completely reconcile with one model or the other, it would be unwise to completely commit oneself to any one theory or model. It is important to look for models which interpret known results and also make predictions that can be verified. Despite the enormous progress made, high temperature superconductivity continues to challenge our intellect.

\section{ACKNOWLEDGEMENTS}

The authors would like to thank the anonymous reviewer for inviting our attention to some recent works that helped improve the objectivity of the manuscript.

\section{REFERENCES}


[1] H. K. Onnes, Comm. Physical Lab. Univ. Leiden No.120b and $122 \mathrm{~b},(1911)$

[2] R. Simon and A. Smith, Superconductors: Conquering Technology's New Frontier (Plenum, New York) 1988

[3] W. Meissner and R. Ochsenfeld, Naturwissen 21, 787 (1933)

[4] F. London and H. London, Proc. Roy. Soc. A149 71 Physica 2, 341 (1935)

[5] V. L. Ginsburg and L. D. Landau, Zh. Eksperim. i Teor. Fiz. 20, 1064 (1950)

[6] J. Bardeen, L. N. Cooper and J.R. Schrieffer, Phys. Rev. 108, 1175 (1957)

[7] C. P. Poole Jr., (ed.) Hand book of Superconductivity (New York Acedemic Press) 2000

[8] C. P. Poole Jr. et al, Superconductivity (New York Academic Press) 1995

[9] J. G. Bednorz and K. A. Muller, Z. Phys. B 64, 189 (1986)

[10] M. K. Wu et al, Phys. Rev. Lett. 58, 908 (1987)

[11] Z. Z. Sheng, Applied Physics Letters 52, 988 (1993)

[12] C. N. R. Rao et al, Nature 326, 856 (1987)

[13] L. Gao et al, Phys. Rev. B 50, 4260 (1994)

[14] L. N. Cooper, Phys. Rev. 104, 1189 (1956)

[15] X. Q. Haung arxiv:1102.1467v1 (2011)

[16] P. Auban-Senzier et al, Synthetic Metals 55-57, 3027 (1993)

[17] J. P. Franck, Physical properties of High Temperature Superconductors IV ed D M Ginsberg (Singapore: World Scientific) 1994

[18] M. K. Crawford et al., Phys. Rev. B 41, 282 (1990)

[19] E. Maxwell, Phys. Rev. 78, 477 (1950)

[20] C. A. Reynolds, B. Serin , W. H. Wright and L. B. Nesbitt, Phys. Rev. 78, 467 (1950)

[21] H. Frolich, Phys. Rev.79, 845 (1950)

[22] M.R. Schafroth, Phys. Rev. 100, 463 (1955)

[23] L. R. Testardi, J. H. Wernick and W. A .Royer, Solid St. commun. 15, 1 (1974)

[24] J. R. Gravaler, M. A. Janocoko and C. R. Jones, J. Applied Phys. 45, 3009 (1974)

[25] S. Fujita and S. Goday, Theory of high Temperature Superconductivity ( Academic Publishers, Kulwer) 2003

[26] P. J. Ford and G. A. Saunder, The Rise of Superconductors ( New York CRC Press) 2005

[27] A. Marouckine, Room Temperature Superconductivity (Cambridge UK Cambridge Science publishing) 2004

[28] C. A. Reynolds, B. Serin and L. B.Nesbitt, Phys. Rev. 84, 691 (1951)

[29] E. A. Lynton, Superconductivity Methuen London (1969)

[30] J. L. Olsen, Cryogenics 2, 356 (1963)
[31] E. Maxwell, Rev. Mod. Phys. 36, 144 (1964)

[32] B. T. Mathias, T. H. Geballe, E. Corenzwit and G. W. Hull Jr., Phys. Rev. 129, 1025 (1963)

[33] E. Bucher and C.Palmy, Phys. Letters 24 A, 340 (1967)

[34] R. E. Fassnacht and J. R. Dillinge, Phys. Rev. Letters 17, 255 (1966)

[35] D. C. Larbalestier, A. Gurevich, D. M. Feldmann and A. Poly anski, Nature insight 414, 368 (2001)

[36] D. G. Hinks, H. Clauss and J. D. Jorgensen, Nature 411, 457 (2001)

[37] R. A. Hein and J. W. Gibson, Phys. Rev. 131, 1105 (1963)

[38] E. Bucher, J. Muller, J. L. Olsen and C. Palmy, Phys. letters $15,303(1965)$

[39] T. H. Geballe, B. T. Mathias, G. W. Hull Jr. and E. Corenzwit, Phys. Rev. Letters 6, 275 (1961)

[40] D. K. Finnemore and D. E. Mapother, Phys. Rev. Letters 9, $288(1962)$

[41] J. W. Gibson and R. A. Hein, Phys. Rev. 141, 407 (1966)

[42] T. H. Geballe and B. T. Mathias, IBM J. Res. Develop 6, 256 (1962)

[43] R. D. Fowler, J. D. G. Linsday, R. W. White, H. H. Hill and B.T.Mathias, Phys. Rev. letters 19, 892 (1967)

[44] N. W. Aschroft and M.Croyt, Europhys. Lett. 23, 605 (1993)

[45] Yu. N. Gartesian et al., Phys. Rev. B 49, 13299 (1994)

[46] A. P. Ramirez et al, Phys. Rev. Lett. 68, 1058 (1992)

[47] T. W. Ebbsen et al, Nature 355, 620 (1992)

[48] B. Stritzker and W. Buckel, Z. Phys. 25, 71 ( 1972)

[49] R. J. Muller and C. B.Scatterthwaite, Phys. Rev. Lett. 34, 144 (1975)

[50] J. A. Schlueter et al, Physica C 265, 163 (1996)

[51] G. M. Eliashberg, Sov-Phy s.-JETP 11, 696 (1960)

[52] W. L. McMillan, Phys. Rev. 167, 331 (1968)

[53] V. J. Emery, Phys. Rev. Lett. 58, 2794 (1987)

[54] P. W. Anderson, Science 235, 1196 (1987)

[55] A. S. Alexandrov and N. F. Mott, Int. J. Mod. Phys. B 8, 2075 (1994)

[56] G. M. Zhao, Physica Scripta 83, 038302 (2011)

[57] K. Yoshida et al, Physica C 156, 481 (1988)

[58] M.K. Crawford et al., Science 250, 1390 (1990)

[59] P. W. Anderson, The theory of Sup erconductivity in High-Tc cuprate sup erconductors (Princeton, NJ: Princeton university Press) 1998

[60] H. J. Boremann, D. E. Morris, H. B.Liu and P. K. Narwankar, Physica C 191, 211 (1992)

[61] J. P. Franck, S.Harker and J. H. Brewer, Phys. Rev. Lett. 71, 
$283(1993)$

[62] G. M. Zhao, K. K. Singh, A. P. B. Sinha and D. E. Morris, Phys. Rev.B 52, 6840 (1995)
[63] G. M. Zhao, K. Conder, H. Keller and K. A. Muller, J. Phy s.: Condens. Matter 10, 9055 (1998)

[64] S. Dal Conte et al Science 335, 1600 (2012) 